\title{
Metastatic nerve root tumor: A case report and literature review
}

\author{
LONG LI, YUAN WU, LIU HU, HONGBIN XU, HAICUI HE and DESHENG HU \\ Department of Radiation Oncology, Hubei Cancer Hospital, Wuhan, Hubei 430079, P.R. China
}

Received December 10, 2015; Accepted March 7, 2016

DOI: $10.3892 / \mathrm{mco} .2016 .852$

\begin{abstract}
Nerve root metastasis of cancer has been rarely reported. We herein report the case of a cervical cancer patient with metastasis to peripheral nerve roots. A 47 year-old woman with cervical squamous cell carcinoma was admitted to our department with a 6-month history of right leg pain, and was investigated for cancer recurrence. Magnetic resonance imaging revealed lymph node metastasis near the right iliac blood vessels; the patient was then treated with chemotherapy with paclitaxel and carboplatin. However, the pain worsened and the muscle strength of her right leg decreased. On positron emission tomography/computed tomography scans, the sacral plexus L5/S1 and L4/5 nerves appeared thickened, suggesting nerve metastases. Intensity-modulated radiation therapy was applied, with notable clinical benefit. However, the patient succumbed to the disease 3 months later.
\end{abstract}

\section{Introduction}

Nerve root metastasis of carcinoma is rare, and no cases of cervical cancer metastatic to the nerves have been reported to date. In reported nerve root metastasis of other tumors, surgical removal of the involved nerves has been performed, whereas treatment with radiotherapy was also recommended (1-3). We herein describe the case of a patient with lumbosacral nerve root metastasis of recurrent cervical cancer. To the best of our knowledge, intensity-modulated radiation therapy (IMRT), was adopted for the first time to treat these lesions.

\section{Case report}

A 47 year-old woman was diagnosed with cervical squamous cell carcinoma in 2013 and underwent radical resection in a country hospital. The patient was confirmed to have stage IIIa disease and received one cycle of adjuvant chemotherapy; however, the details of the adjuvant treatment are not known.

Correspondence to: Professor Desheng $\mathrm{Hu}$, Department of Radiation Oncology, Hubei Cancer Hospital, 116 South Road, Zhuodaoquan, Wuhan, Hubei 430079, P.R. China

E-mail: hds_005@163.com

Key words: cervical cancer, nerve root, metastasis, radiation therapy
The patient declined further treatment due to personal reasons. Six months later, the patient presented to our hospital with pain in her right leg, without dyskinesia. Magnetic resonance imaging (MRI) examination revealed lymph node metastasis near the right iliac blood vessels, involving the right iliac and internal obturator muscles. Subsequently, the patient was treated with 6 courses of chemotherapy with paclitaxel $210 \mathrm{mg}$ and carboplatin $400 \mathrm{mg}$, every 21 days (TP regimen) and chemoradiotherapy (IMRT: Primary gross tumor volume: 55 Gy in 25 fractions; planning target volume: 45 Gy in 25 fractions; and chemotherapy: cisplatin $40 \mathrm{mg} /$ week) for local lesions. However, the pain in the right leg was not alleviated; moreover, the muscle strength decreased, and the patient was nearly unable to walk. The Visual Analog Scale (VAS) pain score was 8 to 9 , the muscle strength of the right leg was level 2+, whereas it was normal in the left leg. Follow-up pelvic MRI (Fig. 1) and whole-body bone scan did not reveal any metastases in the bones or muscles of the right lower limb. Subsequently, positron emission tomography/computed tomography (PET/CT) scan was performed (Fig. 2), revealing sacral plexus L5/S1 and L4/5 nerve thickening, suggesting nerve metastases, which was the cause of the intolerable leg pain.

Oxycodone $60 \mathrm{mg}$ was administered every $12 \mathrm{~h}$ to control the pain and a second radiotherapy was performed for the nerve root metastases. The radiation dose was planned to be 40 Gy in 22 fractions (Fig. 3). However, after delivering 27 Gy (15 fractions), the patient discontinued radiotherapy, since there was no obvious improvement in her symptoms. One month later, the patient experienced a mild pain relief and improved muscle strength of her right leg. The VAS pain score decreased to 5 and the muscle strength increased to level 4 . However, the patient finally succumbed to tumor progression 3 months after the radiotherapy.

The patient's family consented to the publication of the details and radiographic images of this case

\section{Discussion}

Cervical cancer is the second leading cause of cancer-related mortality in women. The recurrence rate for stage III disease is $\leq 42 \%$. Distant recurrence is commonly reported in the following sites: Para-aortic lymph nodes (81\%), lungs (21\%), and supraclavicular lymph nodes (7\%) (4). However, no cases on nerve root metastasis from cervical cancer have been reported to date. Even in other types of cancer, nerve root metastasis is a rare occurrence. Nerve root metastasis has 


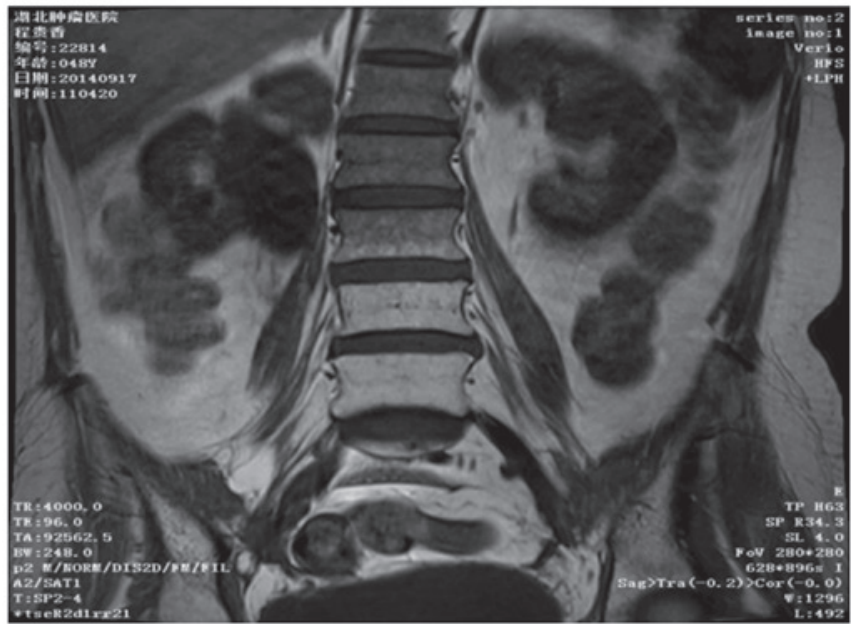

Figure 1. Magnetic resonance imaging of the pelvis. No obvious abnormal signals were seen in lumbar vertebral bodies and their accessories. A cystic shadow was observed in right renal hilum. Lumbosacral nerve plexus were nearly normal.
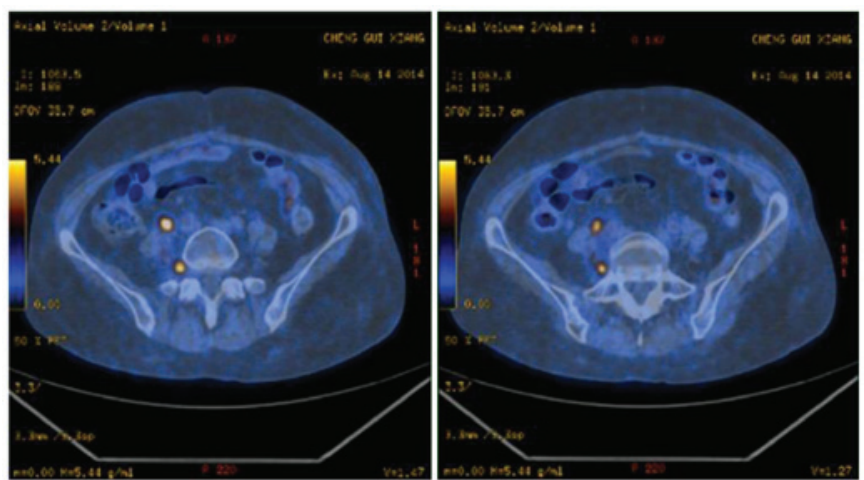

Figure 2. Positron emission tomography/computed tomography scan revealed thickening of the right sacral plexus L5/S1 and L4/5 nerves, which was considered as nerve metastases.

been reported in renal (5), prostate (6) and lung cancer (1), and patients with nerve root metastasis have a very poor prognosis. However, due to the scarcity of such cases, there is limited experience regarding their treatment.

Since MRI is of little value in the detection of nerve root metastasis, it is very easy to miss nerve root metastasis from cancer and delay its diagnosis and treatment (3). Similar to malignant peripheral nerve sheath tumors, $\mathrm{PET} / \mathrm{CT}$ is more sensitive than MRI for detection (7). Thus, in patients with unexplained leg pain, nerve root metastasis should be considered following exclusion of bone metastasis and PET/CT is considered a better choice.

Moreover, the choice of treatment for our patient was complicated. Surgery was employed in the majority of reported cases of nerve root metastasis from cancer (1-3). Following surgery, the pain is usually relieved, but the prognosis remains poor. Radiotherapy may also be another recommendation. The patient in our case refused to undergo surgery. In fact, since the tumor was located at a deep site and her health was poor, it is questionable whether the patient would be able to tolerate a second surgery. Considering the fact that radiotherapy is effective for advanced cervical cancer, we employed radiation

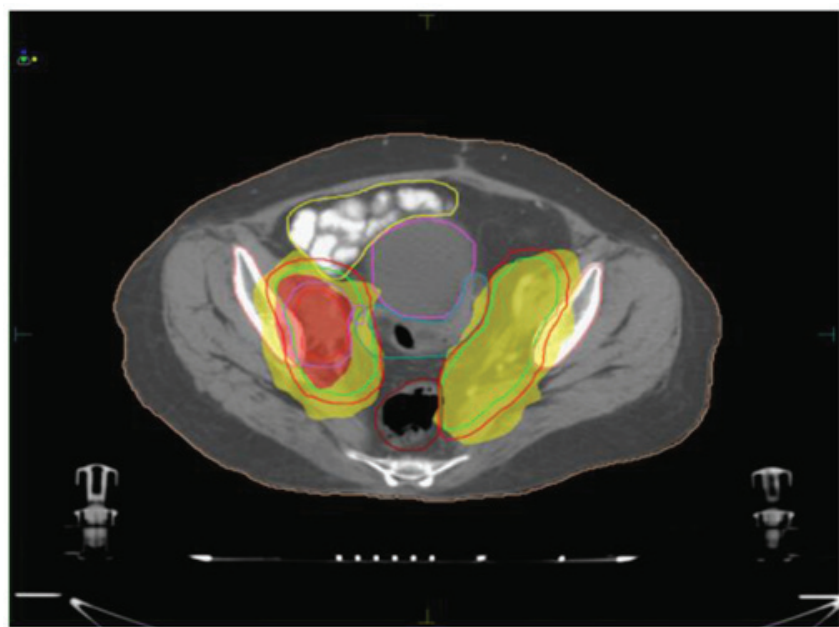

Figure 3. Radiotherapy target figure: Red, gross tumor volume (GTV), which included the involved nerve roots; planned dose to GTV: 40 Gy in 22 fractions. Yellow, clinical tumor volume, which included the high-risk lymphatic drainage area.

treatment for this patient. As it was the second radiation, a palliative dose was administered. However, after 15 fractions of radiotherapy, the patient discontinued treatment. Of note, although the patient did not complete the entire course of radiotherapy, she benefited from this treatment: Her VAS pain score decreased from $>8$ to 5 , while the muscle strength of the right leg increased from level $2+$ to 4 .

In conclusion, our experience suggests that radiotherapy may be a practical choice for nerve root metastasis from cervical cancer, particularly for patients with advanced-stage disease.

\section{Acknowledgements}

The present study was partly supported by NFSC grants (no. 81401913 to Yuan Wu).

\section{References}

1. Slotty PJ, Cornelius JF, Schneiderhan TM, Alexander KM and Bostelmann R: Pulmonary adenocarcinoma metastasis to a dorsal root ganglion: A case report and review of the literature. J Med Case Rep 7: 212, 2013.

2. Strong C, Yanamadala V, Khanna A, Walcott BP, Nahed BV, Borges LF and Coumans JV: Surgical treatment options and management strategies of metastatic renal cell carcinoma to the lumbar spinal nerve roots. J Clin Neurosci 20: 1546-1549, 2013.

3. Uchida K, Kobayashi S, Yayama T, Muramatsu J, Kurokawa T, Imamura Y and Baba H: Metastatic involvement of sacral nerve roots from uterine carcinoma: A case report. Spine J 8: 849-852, 2008.

4. Peiretti M, Zapardiel I, Zanagnolo V, Landoni F, Morrow CP and Maggioni A: Management of recurrent cervical cancer: A review of the literature. Surg Oncol 21: e59-e66, 2012.

5. Cabrilo I, Burkhardt K, Schaller K and Tessitore E: Renal carcinoma relapse presenting as a peripheral nerve sheath tumor: A case report and brief review of the literature. Neurochirurgie 59: 128-132, 2013.

6. Ladha SS, Spinner RJ, Suarez GA, Amrami KK and Dyck PJ: Neoplastic lumbosacral radiculoplexopathy in prostate cancer by direct perineural spread: An unusual entity. Muscle Nerve 34: 659-665, 2006

7. Khiewvan B, Macapinlac HA, Lev D, McCutcheon IE, Slopis JM, Al Sannaa G, Wei $W$ and Chuang HH: The value of ${ }^{18} \mathrm{~F}-\mathrm{FDG}$ PET/CT in the management of malignant peripheral nerve sheath tumors. Eur J Nucl Med Mol Imaging 41: 1756-1766, 2014. 